\title{
The startle response as an indicator of temporal summation
}

\author{
TERRY D. BLUMENTHAL \\ Hamilton College, Clinton, New York \\ and \\ W. KEITH BERG \\ University of Florida, Gainesville, Florida
}

\begin{abstract}
The present study assessed temporal summation of transient and sustained stimuli in the startle eyeblink response system. In two experiments, adult subjects received 95-dB(A), fast-rising broadband noise bursts of two types: (1) single stimuli varying in duration from 20 to 100 msec (Experiment 1) or 30 to $55 \mathrm{msec}$ (Experiment 2) and (2) pairs of 3-msec bursts presented at interpulse intervals corresponding to the single stimulus durations. In addition, a single 3-msec pulse was used as an anchor point for both stimulus types. Though the temporal functions depended on whether startle amplitude or probability was assessed, both measures showed that temporal summation was similar for sustained stimuli and pulse pairs up to about 40 to 50 msec. Beyond this point, single stimuli maintained responding to $100 \mathrm{msec}$, whereas the second pulse of the pair quickly lost its effect. The results indicate that, although startle is influenced by summation of the sustained aspects of a stimulus, summation of transients produces an equivalent effect and does so with more acoustic efficiency (requires less energy). Response latency measures showed no significant summation with paired pulses, and only a narrow summation window for single stimuli. Thus, differential summation of sustained and transient information is demonstrated by all three response measures, but in different ways.
\end{abstract}

The present study was designed to compare temporal summation of transient and sustained auditory stimuli in human adults as reflected by differential activation of the startle response. Stimuli were either pairs of brief stimuli (transients) or analogous single stimuli, with intervals between stimuli in a pair being equal to the durations of the single stimuli.

The processing of sensory input may occur in different ways for transient and sustained stimuli. Brief, transientonly stimuli activate onset-sensitive response systems and determine stimulus detection; sustained stimuli activate response systems that are sensitive to more prolonged stimulus attributes and determine stimulus identification. A stimulus with both transient and sustained properties may activate both types of response system. This transientsustained dichotomy has been found for auditory (Graham, 1979), tactile (Verrillo, 1968), and visual (Schwartz \& Loop, 1984) inputs, and underlying neurological mechanisms have been proposed in each modality (Gersuni, 1971; Gescheider, 1976; Hickey, 1977).

Portions of this research were submitted by the first author to the Graduate School of the University of Florida, in partial fulfillment of the requirements of the degree of Doctor of Philosophy, and were presented at the 24th Annual Meeting of the Society for Psychophysiological Research, Milwaukee, Wisconsin, October 1984. Reprint requests should be addressed to W. Keith Berg, Department of Psychology, University of Florida, Gainesville, FL 32611.
One way in which transient and sustained systems differ is in the interval over which temporal summation occurs-brief in transient systems and longer-lasting in sustained systems. Temporal summation involves the integration of sensory input over time. The effectiveness of a stimulus can be increased by increasing its duration, up to some critical duration, beyond which the response as a function of duration reaches asymptote. Also, a pair of stimuli can be more effective than a single stimulus, and this advantage decreases as interstimulus interval increases. The interval above which further increases in interstimulus interval have no greater effect can be considered a "critical interval," analogous to the critical duration. In the auditory system, temporal summation has been investigated using behavioral scaling (Zwislocki, 1969), single-unit responding (Gersuni, 1971), and brainstem reflex elicitation (Marsh, Hoffman, \& Stitt, 1973). The present study used the acoustic startle reflex to assess temporal summation in human transient and sustained systems.

It has been stated that the acoustic startle response is primarily an indicator of transient system activity (Dykman \& Ison, 1979; Graham, 1979). This may be the case in the rat, but human startle may be influenced by both transient and sustained activity. In the rat, increasing stimulus intensity or decreasing stimulus rise time, both of which affect stimulus onset, causes increases in startle magnitude (Davis, 1974; Fleshler, 1965; Ison, 1978). 
Startle magnitude also increases as stimulus duration increases up to $8 \mathrm{msec}$, but not beyond, illustrating very rapid temporal summation in rats (Fleshler, 1965; Marsh et al., 1973). This rapid summation is also illustrated by the fact that startle amplitude decreases as the interval between two brief $(1-\mathrm{msec})$ pulses increases from 3 to 6 msec; beyond this interval the second pulse does not contribute to the response (Marsh et al., 1973) in rats. In humans, as in rats, increasing stimulus intensity or decreasing stimulus rise time results in larger responses (K. M. Berg, 1973; Blumenthal \& W. K. Berg, 1982). However, temporal summation lasts longer in humans than in rats, as illustrated by K. M. Berg's (1973) finding that response magnitude increases as stimulus duration increases from 1 to $32 \mathrm{msec}$. Also, Yamada (1983) found that startle magnitude increases as stimulus duration increases to $50 \mathrm{msec}$, but not beyond. The duration beyond which summation no longer increases responding (the critical duration) may represent the width of the temporal summation window (the time constant for transient summation), and this may be longer in humans than in rats. The intensity of the stimuli used in startle research is usually higher in studies using rats as subjects than in those dealing with human responding. Although the time constant of transient temporal summation will decrease as stimulus intensity increases, the species differences described above are large enough to suggest a fundamental difference in startle across species. It may be that, although startle in rats is due to transient system activity alone, human startle may reflect both transient and sustained system activity.

To optimally compare the effects of transient and sustained components of stimuli on startle, and to ascertain their temporal summation functions, it would be desirable to obtain measures of each component separately. When sufficiently brief, a stimulus will have only transient characteristics, with no physiologically significant sustained component and no independent second transient at stimulus offset (Green, 1973). In the present studies, 3-msec noise bursts were assumed to produce a single acoustic transient, since peripheral recovery takes longer than $3 \mathrm{msec}$ (Green, 1973).

If one initiates a blink with an onset transient, will there be a further contribution of the sustained portion in a sufficiently long stimulus? This question can be addressed by varying the duration of stimuli with fast rise and fall times, and measuring responding as a function of stimulus duration. The effect of the offset of the sustained stimuli is assumed to be negligible, an assumption that is supported by recent preliminary data (Blumenthal, unpublished data) which suggest that stimulus onset and offset are not equally important for startle. Using stimuli at 80 and $85 \mathrm{~dB}(\mathrm{~A})$, with durations of 30 and $50 \mathrm{msec}$, stimulus rise and fall time were independently varied between 2.5 and $25 \mathrm{msec}$, with adult subjects. Although effects of rise time were significant, no reliable effects of fall time appeared. These stimuli were less intense than those used in the present study, but the findings suggest that the contribution of stimulus offset is minimal compared with that of stimulus onset, at least in the determination of the startle response. Since the first pulse in a pair is assumed to be equivalent to the onset of a single stimulus, and the effect of single-stimulus offset is negligible, any difference in responding to single or paired stimuli can be attributed to the differential effectiveness of the sustained portion of the single stimulus and the second pulse in the pair. Therefore, the second pulse in a pair of pulses may be directly compared with the sustained portion of a single stimulus.

If the sustained component of a single stimulus is more effective than the second transient in an analogous pair of stimuli, we would expect response levels for pulse pairs to be significantly less than those for single stimuli. Thus, if the sustained components of a single stimulus do contribute to startle, the shapes of the temporal functions for single and paired stimuli might differ and, at some points, the function for single stimuli might significantly exceed that for the transient-only pulse pairs. That is, the startle response should be larger or more probable at some duration of the single stimulus than it would be for a pair of stimuli separated in time by an analogous interval.

\section{EXPERIMENT 1}

\section{Method}

Subjects. The subjects were 16 adults (6 males and 10 females) with a mean age of 20 years (range $=18$ to 27 years). They were selected from the pool of introductory psychology university students, and reported no history of hearing loss and no use of medications on the day of testing.

Stimuli. Broadband noise bursts (20 Hz-20 kHz), at $95 \mathrm{~dB}(\mathrm{~A})$, with a rise-fall time of less than $0.1 \mathrm{msec}$ were used. Seven were single, continuous stimuli varied in duration $(3,20,35,50,65$, 80 , and $100 \mathrm{msec}$ ), and six were pairs of $3-\mathrm{msec}$ pulses at stimulus onset asynchronies corresponding to the $20-100-\mathrm{msec}$ singlestimulus durations. Intertrial interval ranged from 25 to $35 \mathrm{sec}$ and averaged $30 \mathrm{sec}$.

Apparatus. Stimuli were produced by a Grason-Stadler $455 \mathrm{C}$ noise generator gated through an Iconix electronic switch and a Sansui AU517 amplifier and presented through a JBL Decade 26 loudspeaker located approximately $1.5 \mathrm{~m}$ in front of the subjects. Stimulus intensity was calibrated using a General Radio $1551 \mathrm{C}$ sound-level meter and monitored with a Hewlett-Packard $400 \mathrm{E}$ ac voltmeter.

Reflex eyeblink responses were assessed from periorbital electromyographic (EMG) activity collected using miniature Beckman biopotential electrodes $(\mathrm{Ag} / \mathrm{AgCl})$ filled with synapse conducting paste. The EMG signal was amplified by a Coulbourn Hi-Gain Bioamplifier/Coupler with filters passing frequencies of $90-250 \mathrm{~Hz}$. The signal was then integrated with a Coulbourn Contour Following Integrator at a time constant of 80 msec. ${ }^{1}$ The integrated EMG was then recorded on a Beckman R411 polygraph and digitally sampled (10-bit accuracy) by a PDP-8 computer every millisecond for 250 msec after stimulus onset.

Procedure. The experimenter explained the procedure, and the subject was asked to read and sign an informed consent and to fill out a background questionnaire. The experimenter then cleaned the area just below the subject's left eye with a cotton swab soaked in alcohol, and two electrodes were attached, one below the center of the eye and the other immediately temporal to the first, as close to the orbital ridge as possible without impairing eye movement. The subject was then seated in the testing room and asked to move 
as little as possible, expecially the eyes and head. Data were used only from trials on which an experimenter, watching the subject on closed-circuit television, judged the subject's eyes to be open. If a stimulus was presented when the eyes were closed, or during movement of the head or eyes, the trial was rejected by an experimenter blind to stimulus conditions. An average of $7.5 \%$ of the trials were rejected for each subject; rejection was not significantly more likely for one stimulus condition than for any other. A $13 \times 13$ Latin square was used to determine stimulus order, with the row of entry into the square being determined randomly. A session was terminated when six potentially scorable responses in each stimulus condition were obtained.

Data Analysis. Response amplitude was measured as the difference between response onset and peak. Response onset was judged only during the window between 20 and $100 \mathrm{msec}$ after stimulus onset, to limit the analysis to stimulus-elicited reflex eyeblinks only. Initiation of a respose was judged on the basis of a monotonic increase in integrated EMG that continued for at least $20 \mathrm{msec}$. Onset latency was then judged as the point at which this increase exceeded the random variability in the $20 \mathrm{msec}$ prior to this increase. If the slope of the integrated EMG deviated from 0 for at least $20 \mathrm{msec}$ prior to response onset, the trial was rejected, due to the absence of a stable preresponse baseline. Since stimuli were not presented during movement of the head or eyes, these baseline problems were minimal. The peak of the response was the first point following onset at which the slope of the integrated EMG signal equaled or passed through 0 , when this was followed by at least $10 \mathrm{msec}$ with no further slope reversal. Response latency was measured as the time from stimulus onset to response onset.

Response probability was measured as the degree to which responses actually occurred when subject variables were optimal (i.e., eyes open, no head movement). If the integrated EMG signal during the response window did not deviate beyond the random noise present prior to the window, a failure to respond was recorded. Due to the considerable sensitivity of the computer sampling and amplifier, the degree to which very small responses were not detected was minimal.

The response parameter most often reported by researchers in this area is average magnitude, which is calculated from trials on which a response could have been recorded, whether a response actually occurred or not (e.g., Graham \& Murray, 1977). This means that trials on which a response could have occurred but did not are assigned a magnitude score of 0 , which is then included in the calculation of the average magnitude. Magnitude changes can be due to changes in either response probability or amplitude (Prokasy \& Ebel, 1967), and these two may be partially independent measures of startle responding (Blumenthal \& W. K. Berg, in press). Therefore, the present study examined response amplitude and probability separately. For each subject, response amplitude and latency were averaged across trials for each condition. ANOVAs included stimulus type (single or paired) and time (duration or interval) as within-subject variables. To balance the analyses, data from the 3-msec single-stimulus condition were duplicated for use in paired stimulus comparisons, as a substitute for a pair of pulses at an interval of 0 msec.

\section{Results}

Amplitude. Increasing the duration of single stimuli or the interval between pulses in a pair had different effects, as indicated by a significant interaction of stimulus type and time $[F(6,90)=8.88, p<.001]$ (see Figure 1). When pulse pair data were assessed alone, the change in interpulse interval produced a significant quadratic trend $[F(1,15)=5.61, p<.05$.$] More specifically, when the$ interval of pulse pairs was increased to 20 and $35 \mathrm{msec}$, but not beyond, the responses were larger than those ob-

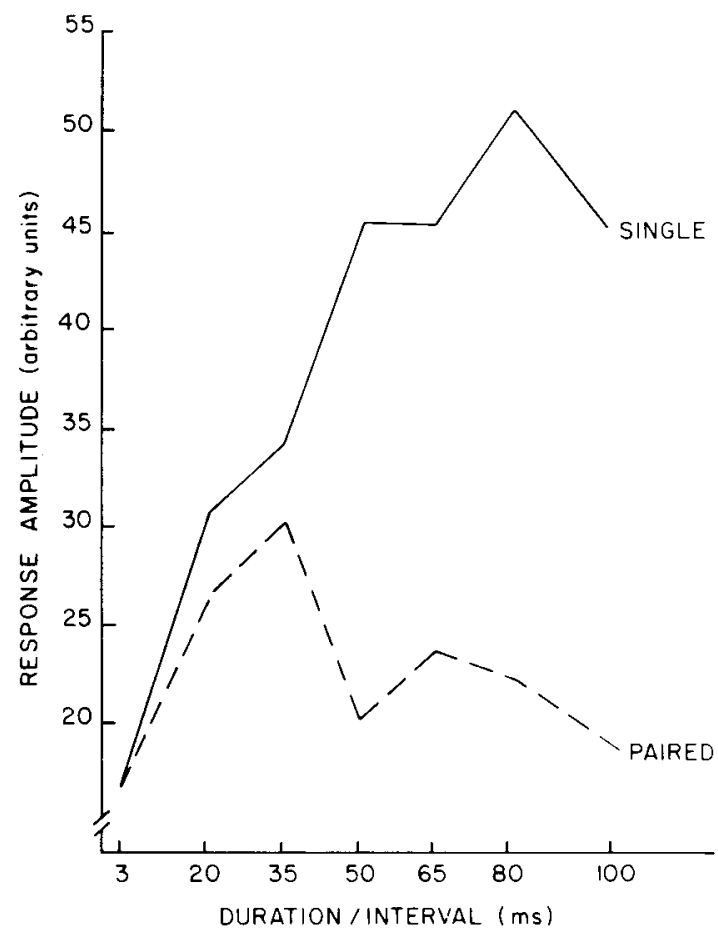

Figure 1. Response amplitude as a function of time, for single and paired stimuli.

tained for a single 3 -msec pulse $[t \mathrm{~s}(15)=2.20$ and 2.85 , respectively, $p<.05$ ], although the responses to the 20 and 35-msec pairs did not differ from each other. At interpulse intervals of $50 \mathrm{msec}$ or more, responses were not significantly larger than they were for a single 3-msec pulse. Increasing single-stimulus duration to $50 \mathrm{msec}$, but not beyond, resulted in larger responses, as shown by significant linear $[F(1,15)=18.23, p<.001]$ and quadratic $[F(1,15)=15.37, p<.001]$ trends. Responses were larger at 20 than at $3 \mathrm{msec}$ duration $[t(15)=4.13, p<$ $.001]$ and larger at 50 than at $35 \mathrm{msec}$ duration $[t(15)=$ $2.64, p<.025]$. No other successive pairwise comparisons reached significance, indicating that increasing duration above $50 \mathrm{msec}$ had no further significant effect. Comparisons across stimulus type at corresponding temporal values indicated that a single stimulus was not significantly more effective than a pair of transients, up through $35 \mathrm{msec}$, but single stimuli were more effective above $35 \mathrm{msec}$.

Probability. The effects of single-stimulus duration and pulse pair interval differed for response probability, resulting in a significant interaction of stimulus type and time $[F(6,90)=5.34, p<.001]$ (see Figure 2). For paired stimuli, a quadratic trend appeared $[F(1,15)=6.16, p<$ $.025]$, but pairwise comparisons failed to show any significant differences between any paired stimuli and a single 3-msec stimulus. Probability was not significantly different for intervals of 20,35 , or $50 \mathrm{msec}$. At longer intervals, the slight contribution of the second pulse disappeared. For single stimuli, a linear effect of duration 


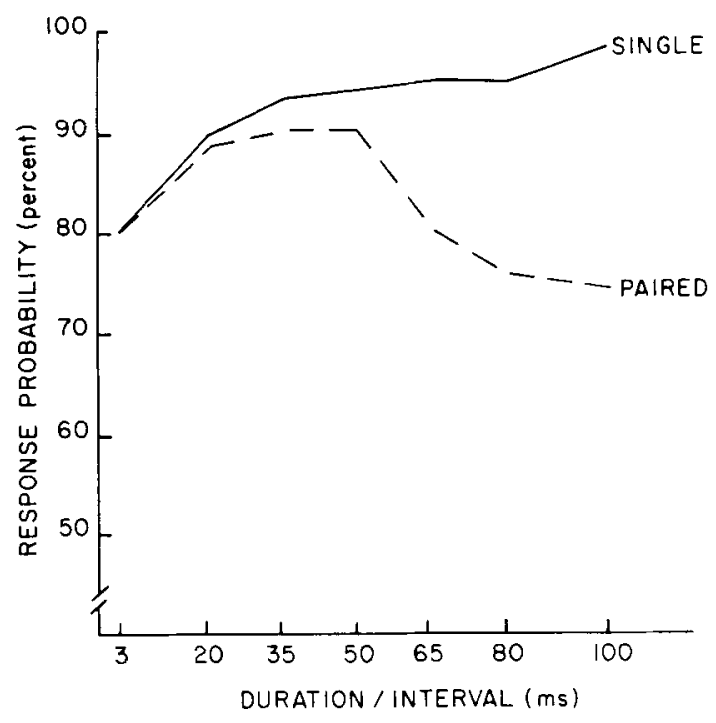

Figure 2. Response probability as a function of time, for single and paired stimuli.

appeared $[F(1,15)=13.29, p<.01]$, and this was due mainly to increasing duration from 3 to $20 \mathrm{msec}[t(15)$ $=2.22, p<.05]$. In fact, if the 3-msec data are excluded from the analysis, no significant duration effect is found. Up to $50 \mathrm{msec}$, the difference in probability for single and paired stimuli was not significant, although this difference was significant beyond $50 \mathrm{msec}$. In the range in which a second transient was not even marginally effective, the sustained portion of the single stimuli maintained a high response probability. However, the interpretation of these probability data may be influenced by a ceiling effect for single stimuli.

Latency. In all cases response latency was longer for pairs of transients than for single stimuli (see Figure 3). The main effect of time on response latency was significant $[F(6,90)=2.22, p<.05]$, as was the main effect for stimulus type $[F(1,15)=10.40, p<.01]$. Although the stimulus type $\times$ time interaction was not significant, the effect of time was looked at for each stimulus type to allow for a comparison with amplitude and probability data. For paired stimuli, increasing interpulse interval had no significant effect on response latency. The effect of single-stimulus duration was significant $[F(6,90)=4.43$, $p<.001$ ], but this was due to the increase from 3 to $20 \mathrm{msec}$ only, since, if the $3-\mathrm{msec}$ data are not included in the analysis, no duration effect is found.

\section{EXPERIMENT 2}

Taken together, the measures of response amplitude, probability, and latency suggest that the temporal summation window for transient stimuli is somewhat longer for response amplitude and probability $(35-50 \mathrm{msec}$ ) than for response latency (less than $20 \mathrm{msec}$ ). To more ac- curately assess responding in the critical range of 35-50 msec, a second experiment was conducted in which stimulus duration and interpulse intervals in this range were varied in 5-msec steps.

\section{Method}

The subjects were 12 university students ( 6 males and 6 females) with an average age of 22 years (range $=18$ to 29), selected in the same way as in Experiment 1. Stimuli, apparatus, and procedure were identical to those used in Experiment 1, except that stimulus durations were $3,30,35,40,45,50$, and $55 \mathrm{msec}$, with analogous interpulse intervals for paired $(3-\mathrm{msec})$ stimuli.

\section{Results}

The results generally replicated those of the first study. Response amplitude increased as stimulus duration for single stimuli and interpulse interval for paired stimuli increased to $40 \mathrm{msec}$, with the single-stimulus function reaching asymptote and the paired-stimulus function dropping off above this point. The difference between the two types of stimuli was significant only at 50 and $55 \mathrm{msec}$. For response probability, increasing stimulus duration to $30 \mathrm{msec}$ resulted in higher probability, but increases beyond this point had no further effect. A pair of pulses at the 30-msec interval was more effective than a single 3msec stimulus, and the probability function for paired stimuli was level until $45 \mathrm{msec}$, above which it dropped off. Single stimuli were no more effective than paired stimuli below $40 \mathrm{msec}$, but were more effective above $40 \mathrm{msec}$. Response latencies for paired stimuli were significantly longer than those for single stimuli, at all intervals tested.

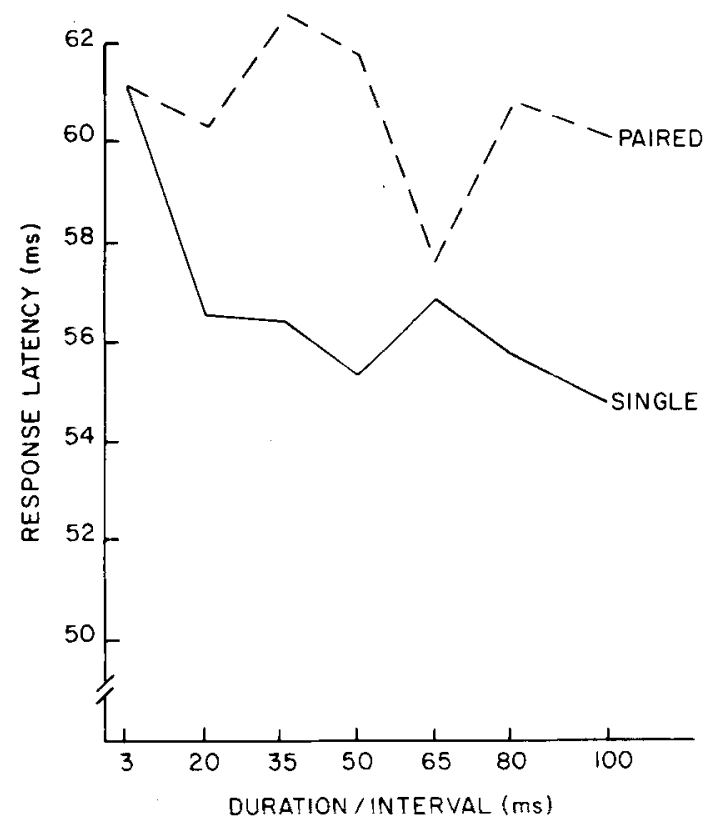

Figure 3. Response latency as a function of time, for single and paired stimuli. 


\section{DISCUSSION}

The findings of the present study suggest that, in determining startle responding, temporal summation of transient acoustic input is more efficient than temporal summation of sustained input. Transients that occur within a window of less than 50 msec summate, and sustained summation adds little that cannot be explained by transient summation in this range. Beyond this window, transient summation does not occur, and the contribution of sustained summation becomes apparent. Sustained summation and transient summation may occur at the same time, but the latter overshadows the former in determining startle responding. Also, these differential summation effects are reflected by startle response amplitude, probability, and latency in different ways.

Two transients are more effective than one, if they occur within a brief enough time, illustrating temporal summation of transients. For response amplitude, transient summation occurs within a window of about $40 \mathrm{msec}$, and the time between transients has a slight, but not significant, effect on the response. As interpulse interval within the window increases, a slight increase in response amplitude may result due to escape from forward masking. In forward masking, the first pulse decreases the effectiveness of the second, and this effect is more pronounced the closer they are in time. As the interpulse interval increases, the amount of masking decreases (Raab, 1961), producing either less masking or no masking, as shown by our finding of a somewhat larger response at the 35msec interval than at the 20-msec interval. Forward masking effects are minimal for very brief maskers (Zwislocki, 1978), so the influence of masking may not be very large in the present study. Also, this marginal masking effect was found for response amplitude, but not for response probability.

For response probability, the effect of the second transient was not influenced by its location within the 50 -msec window. For response latency, the second transient had no significant effect at any of the intervals used. It is possible that, for latency measures, the temporal summation window for transients is narrower than $20 \mathrm{msec}$, so that the second pulse always occurred outside the window; this prediction could be evaluated by future research.

The extent to which the second pulse in a pair elicited a response when the first pulse failed to do so was minimal (occurring on less than 10 trials for all subjects combined). These responses were not included in any data analyses. At long intervals, this was seen as a response with a latency of the interval length plus about $50 \mathrm{msec}$. The second pulse would be even less independently effective at short intervals, due to masking and refractoriness caused by the first pulse. This means that the second pulse alone was generally not effective in eliciting responding.

Within the transient summation window, a second transient was as effective as the sustained portion of a cor- responding single stimulus. This illustrates an asymmetrical effect on startle, since the single stimuli contained much more energy than the pairs of transients. Temporal summation of transients seems to be more efficient than temporal summation of energy (sustained summation). Beyond the transient summation windows $(40 \mathrm{msec}$ for response amplitude, $50 \mathrm{msec}$ for response probability), the second stimulus in a pulse pair had little or no effect, but responding was increased or maintained by the sustained portion of a single stimulus.

The upper limit of the transient summation window is the point beyond which a second transient does not contribute to the response. If a single stimulus causes a further increase in responding beyond this point, this must be due to sustained summation's continuing to increase while transient summation returns to the level determined by stimulus onset only. This is true for response amplitude, so, for this response measure, the window for sustained summation $(50 \mathrm{msec})$ is wider than the window for transient summation $(35 \mathrm{msec})$. On the other hand, if the single stimulus maintains, but does not increase, responding beyond the transient summation window, this demonstrates no increase in the occurrence of sustained summation beyond the transient summation window. This is true for response probability, where the first $20 \mathrm{msec}$ of a single stimulus is sufficient to achieve peak probability, since a 20 -msec stimulus is no less effective than any of the longer stimuli. The transient summation window is $50 \mathrm{msec}$ for response probability, but sustained summation peaks within $20 \mathrm{msec}$ of stimulus onset. However, the possible existence of a ceiling effect limits the interpretation of these probability data.

Graham, Strock, and Zeigler (1981) suggest that a neurological dichotomy of transient and sustained sensory neurons exists. Two types of neurons, which differ in their characteristic discharge patterns to stimuli, have been identified in the auditory pathway (Gersuni, 1971; Radionova, 1971). Short-time-constant (STC) units illustrate a transient discharge at stimulus onset (and at offset, in some cases), whereas long-time-constant (LTC) units discharge in a sustained fashion throughout the duration of the stimulus. These units are also sensitive to different stimulus parameters, with LTC units being sensitive to stimulus duration, but not to rise time, and the opposite being true of STC units. The two types of units show different patterns of temporal summation, with STC units having very brief critical durations (a few milliseconds) and LTC units having much longer critical durations. These units are found at all levels of the auditory pathway, and may be organized into functional systems, resulting in differential processing of transient and sustained stimulus attributes. In this respect, the LTC and STC systems are analogous to Pacinian and non-Pacinian systems in tactile processing (Gescheider, 1976; Verrillo, 1968), and to chromatic (X-cell) and achromatic (Y-cell) systems in visual processing (Schwartz \& Loop, 1984). All three 
modalities illustrate distinctive transient and sustained systems which possess different temporal summation properties.

In a another report (Blumenthal \& W. K. Berg, in press), it was suggested that startle amplitude and probability might be determined by partially independent underlying mechanisms, a startle "amplifier" and a startle "trigger." This is analogous to the distinction made in cognitive perceptual research between stimulus detection and stimulus identification (Posner, 1978), in that the startle trigger may operate to detect the presence of an effective startle stimulus and the startle amplifier then determines the size of the response based on the relevant stimulus parameters. Some evidence suggests that the proposed trigger and amplifier mechanisms may be found either in the auditory pathway below the inferior colliculus or in the startle center itself (Davis, Gendelman, Tischler, \& Gendelman, 1982). Also, the two mechanisms may have different locations, since they appear to be partially independent in their responding. If either the trigger or the amplifier is located in the auditory pathway, similar mechanisms may be found in other sensory pathways, and further studies investigating startle trigger and amplifier mechanisms in visual and tactile modalities are suggested. The fact that analogous temporal summation abilities are found across modalities (Gersuni, 1971; Gescheider, 1976; Schwartz \& Loop, 1984) suggests that this summation may be a very useful means of investigating startle trigger and amplifier mechanisms in several modalities. It would also be useful to conduct studies using animal models, to more accurately locate the mechanisms underlying these findings. These studies may find that the trigger and amplifier mechanisms are due to specific "startle center" involvement, or to basic organizing principles found in all sensory systems.

The trigger may be more sensitive than the amplifier, since response probability peaks at $20 \mathrm{msec}$ for both single and paired stimuli, whereas response amplitude continues to increase until about $40 \mathrm{msec}$ for paired stimuli and about $50 \mathrm{msec}$ for single stimuli. This implies that the amount of energy required to reach maximal activation is lower for the trigger than for the amplifier. In fact, this critical level may be below $20 \mathrm{msec}$, and further research at durations and intervals of less than $20 \mathrm{msec}$ is needed to measure the transient sensitivity of the startle trigger.

As reflected by startle responding, temporal summation appears to have a much longer time constant, for both transient and sustained stimulation, in the human than in the rat. Marsh et al. (1973), using single and paired stimuli with rats, found a critical duration of $4-8 \mathrm{msec}$ and a critical interval for transient summation of $6 \mathrm{msec}$. These findings are analogous to our findings in humans, except for a difference in the time constants found. The similarities in human and rat startle responding to transient and sustained stimuli, given different absolute time courses, supports the use of nonhuman research to model human temporal summation.

The present study shows that the human startle response is influenced by activity in both the transient and sustained systems. These two systems are at least partially independent, with the startle response being the net result of activity in both systems. However, the relative contribution of these two systems to the startle response may not be equal, since our results suggest that the transient system is more efficient than the sustained system. Also, the differential effects of transient and sustained stimuli on startle response amplitude, probability, and latency support the partial independence of these response measures, possibly reflecting different underlying mechanisms.

\section{REFERENCES}

BERG, K. M. (1973). Elicitation of acoustic startle in the human. (Doctoral dissertation, University of Wisconsin, 1973). Dissertation Abstracts International, 34, 5217B-5218B. (University Microfilms No. 74-3510,99).

Blumenthal, T. D., \& Berg, W. K. (1982). Developmental differences in startle response to stimulus onset transition. Psychophysiology, 19, 551-552. (Abstract)

Blumenthal, T. D., \& BeRG, W. K. (in press). Stimulus rise time, intensity, and bandwidth effects on acoustic startle amplitude and probability. Psychophysiology.

DAvis, M. (1974). Signal-to-noise ratio as a predictor of startle amplitude and habituation in the rat. Journal of Comparative \& Physiological Psychology, 86, 812-825.

Davis, M., Gendelman, D. S., Tischler, M. D., \& Gendelman, P. M. (1982). A primary acoustic startle circuit: Lesion and stimulation studies. Journal of Neuroscience, 2, 791-805.

Dykman, B. M., \& Ison, J. R. (1979). Temporal integration of acoustic stimulation obtained in reflex inhibition in rats and humans. Journal of Comparative \& Physiological Psychology, 93, 939-945.

FLESHLER, M. (1965). Adequate acoustic stimulus for startle reaction in the rat. Journal of Comparative \& Physiological Psychology, 60, 200-207.

GERSUNI, G. V. (1971). Temporal organization of the auditory function. In G. V. Gersuni (Ed.), Sensory processes at the neuronal and behavioral levels. New York: Academic Press.

Gescheider, G. A. (1976). Evidence in support of the duplex theory of mechanoreception. Sensory Processes, 1, 68-76.

GraHam, F. K. (1979). Distinguishing among orienting, defense, and startle reflexes. In H. D. Kimmel, E. H. van Olst, \& J. F. Orlebeke (Eds.), The orienting reflex in humans. Hillsdale, NJ: Erlbaum.

Graham, F. K., \& Murray, G. M. (1977). Discordant effects of weak prestimulation on magnitude and latency of the reflex blink. Physiological Psychology, 5, 108-114.

GrahaM, F. K., STrock, B. D., ZEIGLER, B. L. (1981). Excitatory and inhibitory effects on reflex responsiveness. In W. A. Collins (Ed.), Aspects of the development of competence. Hillsdale, NJ: Erlbaum.

GrEeN, D. M. (1973). Minimum integration time. In A. R. Moller (Ed.), Basic mechanisms in hearing. New York: Academic Press.

HiCKEY, T. L. (1977). Postnatal development of the human lateral geniculate nucleus: Relationship to a critical period for the visual system. Science, 198, 836-838.

IsON, J. R. (1978). Reflex inhibition and reflex elicitation by acoustic stimuli differing in abruptness of onset and peak intensity. Animal Learning \& Behavior, 6, 106-110.

MarSh, R., Hoffman, H. S., \& STITT, C. L. (1973). Temporal integration in the acoustic startle reflex of the rat. Journal of Comparative \& Physiological Psychology, 82, 507-511. 
PosNer, M. I. (1978). Chronometric explanations of mind. Hillsdale, NJ: Erlbaum.

Prokasy, W. F., Ebel, H. C. (1967). Three components of the classically conditioned GSR in human subjects. Journal of Experimental Psychology, 73, 247-256.

RAAB, D. H. (1961). Forward and backward masking between acoustic clicks. Journal of the Acoustical Society of America, 33, 137-139.

Radionova, E. A. (1971). Two types of neurons in the cat cochlear nuclei and their role in audition. In G. V. Gersuni (Ed.), Sensory processes at the neuronal and behavioral levels. New York: Academic Press.

SchwarTz, S. H., LOOP, M. S. (1984). Effect of duration on detection by the chromatic and achromatic systems. Perception \& Psychophysics, 36, 65-67.

VERRILLO, R. T. (1968). A duplex mechanism of mechanoreception. In D. R. Kenshalo (Ed.), The skin senses. Springfield, IL: Thomas.

YAMADA, F. (1983). Effects of intensity, duration and rise and fall times of the reflex evoking sound upon auditory evoked EMG of orbicularis oculi and subjective estimation of startle. Japanese Journal of Physiological Psychology \& Psychophysiology, 1, 11-18.
ZWISLOCKI, J. J. (1969). Temporal summation of loudness: An analysis. Journal of the Acoustical Society of America, 46, 431-441.

ZwISLOCKI, J. J. (1978). Masking: Experimental and theoretical aspects of simultaneous, forward, backward, and central masking. In E. C. Carterette \& M. P. Friedman (Eds.), Handbook of perception: Vol. 4. Hearing. New York: Academic Press.

\section{NOTE}

1. The integrator time constant was determined by direct measure ment of the exponential recovery function of a known pulse input, not by the front panel setting on the integrator itself. It was found that the specified time-constant setting of the integrator was not accurate, so the only reliable way to determine the actual time constant was by calibration.

(Manuscript received November 11, 1985; revision accepted for publication May 27, 1986.) 